steht, welche zu den bekannten streifigen Verdünnungen dèrselben führt; und wodurch die Epidermisdecke einreisst, nichts desto weniger Finger* drücke zurückbleiben seben, sondern insbesondere daraus, dass die Spannung der Haut hier nur eine mässige war. -

Ich bin weit davon entfernt, zu glauben, dass ich durch vorstehende Erörterungen die Besonderheiten, welche die Hautwassersucht im vorliegenden Falle von Hautwassersucht bei diffuser Nephritis darbot, erklärt habe. Die Mittheilung der Thatsache schien mir der Erwähnung wert. Was Barteis in seinen vortrefflichen Nieren-Krankheiten (v. Ziems s en's Sammelwerk der spec. Pathol. u. Therap. 9, I, Leipzig 1875, S. 97) betreffs der Hautwassersucht bei Nierenkrankheiten sagt: „dass bei der Localisation dieser Wassersucht, abgeseben von der Gravitation, noch sonstige Umstände in Betracht kommen, welche sich nicht in jedem Falle eruiren lassen", besteht auch heute noch zu Recht. Wir werden uns dadureh aber nicht abhalten lassen dürfen, die Beobachtungen vorläufig zu registriren. Meine Absicht, Oedemflüssigkeit ans der qu. Localität zu untersuchen und sie mit der an anderen Körperstellen zu vergleichen, war nicht möglich, denn, wie sich aus der Krankengeschichte ergiebt, war durch die Punction keine Oedemflüssigkeit zu erhalten.

2.

\title{
Beitrag zur Lehre vom Zittern $\left.{ }^{1}\right)$.
}

\author{
Von Dr. Levy-Dorn in Berlin.
}

Unter Zittern verstehen wir unwillkürliche, einigermaassen rhytbmische, schnell aufeinanderfolgende Bewegungen desselben Gliedes. Da die bewegende Kraft von den Muskeln stammt, scheint es genauer in die Begriffsbestimmung statt "Glied“ das Wort "Muskel" zu setzen. Doch ich möchte davon abrathen, da wir sonst das sog. fibrilläre Zittern, welches von den übrigen Arten des. Tremors wesentlich abweicht und deshalb besser mit Muskelfimmern bezeichnet wird, in unsere Definition einbeziehen müssten.

Auch für das Wort „Muskel" „Muskelgruppen“ zu setzen, wie es von einzelnen Autoren geschieht, geht nicht an. Denn einmal können auch Muskelgruppen fibrillär zucken, ohne dass es zum eigentlichen Tremor kommt, andrerseits ist noch nicht widerlegt, dass der Tremor unter Um-

1) Nach einem, am 9. Mai 1898 in der Berliner Gesellschaft f. Psychiatrie und Nervenkrankheiten gehaltenen Vortrage. 
ständen von einem einzigen Muskel erzeugt werden kann, dessen Antagonist etwa durch ein elastisches Band vertreten wird.

Dass aber einem jeden Tremor eine mebr oder weniger rbythmische Bewegung des Muskels zu Grunde liegt, obwohl nicht alle solche Bewegungen Zittern hervorrufen $\mathrm{m} u ̈$ ssen, unterliegt natürlich keinem Zweifel.

Das Wesen eines pathologischen Vorgangs erscheint uns verständlicher, wenn wir ein physiologisches Analogon an seine Stelle setzen können.

Wir haben nun bekanntlich in jedem tetanisch contrahirten Muskel eine zwar nicht ohne Weiteres sichtliche, aber doch durch sorgfâltige Analyse sicber nach weisbare Vielheit von Zuckungen. Ich brauche nur an den Muskelton und den secundären Tetanus zu erinnern: Der durch den Willen tetanisirte Muskel bleibt nicht beständig auf seiner "Hubhöhe*, sondern vibrirt um sie berum, der Tetanus ist, wie man sagt, ein unvollkommener.

Das Experiment lehrt, dass die Erscheinung darauf beruht, dass die Schnelligkeit, mit welcher die Einzelreize den Muskel treffen, eine gewisse Grösse nicht übersteigt. Die gegebenen Curven, welche ich dem Lehrbuch der Physiologie des Menschen von Gad und Heymans entnehme, mögen das Wesen der besprochenen Vorgänge veranschaulichen:

a)

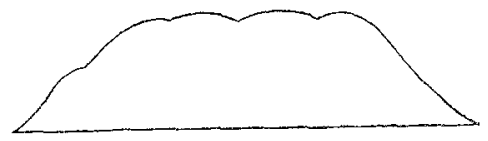

b)

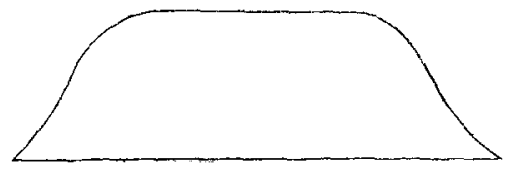

Tetanus desselben Muskels

a) unvollkommener bei 20 maximalen Einzelreizen in der Secunde.

b) vollkommener bei 40 Einzelreizen in der Secunde.

Die Zahl der Schwankungen, denen der willkürlich contrahirte Muskel unterliegt, wurde von Helmboltz auf 19,5 in der Secunde festgesetzt. Die von ihm angewandte Methode, die Bestimmung des Muskeltons, ist aber nicht ganz einwandsfrei. Spätere Untersuchungen mit Hülfe von Registrir-A pparaten, wie Uebertragungen der Bewegung durch M a r e y'sche Trommeln, in die Muskeln gesteckte Nadeln, in die Fasern gebrachte Häkchen oder Capillar-Electrometer zeigten, dass jene Zahl zu boch ausgefallen war. Thatsächlich liegt sie um 10 herum.

Es ist bekannt, dass sich die Zitterschwankungen häufig mit derselben Geschwindigkeit. wiederholen, und es besteht also insofern eine 
bemerkenswerthe Uebereinstimmung zwischen dieser pathologischen Bewegung und dem normalen Tetanus.

Eine andere Aehnlichkeit zwischen beiden Erscheinungen liegt in ihrem nervösen Ursprung. Denn die bei Kranken beobachteten Zitterarten sind ebensowenig, wie die willkürliche Contraction ohne Vermittlung des Centralnerven-Systems denkbar.

Es entsteht nun die Frage, welehem Theil des Nervensystems der wesentlichste Antheil am Zustandekommen jener beiden discontinuirlichen Bewegungen gebührt.

Horsiey und Schaefer (Journ. of physiol VII. 1886) studirten diese Frage für die normalen Muskelcontractionen. Sie fanden bei Reizung der Hirnrinde, wie bei spontanen und reflectorischen Bewegungen, bei Reizung der Corona radiata, wie des Rückenmarks wesentlich denselben Rhythmus der Bewegung, nehmlich 8-13, meist 10 Vibrationen in der Secunde. Bei Reizung des Nerven entspricht die Zahl der Muskelschwankungen derjenigen der Reizfrequenz, wenn diese nicht so hoch wird, dass vollkommener Tetanus eintritt. Bei epileptoiden Bewegungen beobachtet man oft ein Verscbmelzen einzelner Wellen zu einer grösseren. Es bleibt in der citirten Arbeit unentschieden, ob die Discontinuität der Bewegung in der Hirnrinde zu Stande kommt, weil Reizung der Medulla oblongata und des Rückenmarks, also der untergeordneten Centren, dieselbe Wirkung hat, wie Reizung der Rinde. Es wäre ja immerhin denkbar, dass an sich continuirliche Impulse der Rinde dureh die Ganglien niederer Ordnung - wie etwa der constante Strom durch den Wagner'schen Hammer rhythmisch unterbrochen werden.

Es giebt aber einen anderen Weg, zu prüfen, ob in der That die Rinde so schnell hintereinanderfolgende Reize auszusenden vermag, wie wir sie beim Tetanus und Tremor beobachten, und da so mancherlei für den Sitz wenigstens einiger Tremorarten in der Rinde spricht, so wollen wir jenen Weg betreten.

Die physiologische Psychologie verlegt die sogenannte willkürliche oder bewusste Handlung in die höchsten Sphären des Nervensystems. Wir brauchen also, um die aufgestellte Frage zu lösen, nur nachzusehen, wie oft eine willkürliche Bewegung hintereinander wiederholt werden kann.

Ich liess einen elektrischen Contact durch abwechselndes Heben und Senken des Zeigefingers schliessen und offnen und forderte die Versuchsperson auf, die Bewegung möglichst schnell zu wiederholen. Ein RegistrirApparat zeichnete den Vorgang auf eine Kymographion-Trommel, in weleher in herkömmlicher Weise das Resultat abgelesen wurde. Zum Schliessen and Oeffnen des Stromes diente der bei Telegraphen-Einrichtungen gebräuchliche Morse-Schlüssel, zur Uebertragung auf die berusste Fläche der Trommel ein Pfeil'sches Signal.

Eine Reihe von Versuchen, die ich an sechs verschiedenen gesunden Personen ausführte, ergab, dass der Finger in einer Secunde höchstens Archiv f. pathol. Anat. Bd. 155. Hft. 3. 
9-11 mal hin- und herbewegt werden konnte. Die Zahl 9 wurde verhältnissmässig leicht erreicht. Die Zahl 11 wurde nur bei einem Individuum gezählt. Ein sehr kräftig gebauter, in Sport und athletischen Künsten geübter Mann aber brachte bei grösster Anstrengung nie mehr, wie 7 Bewegungen in der Secunde hervor.

Es ist sehr wahrscheinlich, dass die Uebung und die Art der körperlicben Ausbildung eine gewisse Rolle für die fraglicbe Leistung spielt. Doch babe ich meine Untersuchungen nach dieser Richtung bin nicht abgeschlossen.

Die bedeutend höheren Geschwindigkeiten, wie sie scheinbar bei einzelnen Hantirungen, z. B. beim Spielen eines Instrumentes, erreicht werden beruhen auf dem gesehickten Gebrazch mehrerer Finger hintereinander. Bevor der eine Finger seine Bewegung vollständig vollbracht hat, fallen die anderen Finger der Reihe nach zu demselben Zweck ein und lösen ihn ab. Dadurch, dass ich zuerst den Knopf des Morse-Schlüssels mit dem kleinen Finger niederdrückte, ihn erbob, dasselbe mit dem vierten und Wweiten Pinger hintereinander that, erzielte ich scheinbar eine 4 mal so schnelle Bewegung, als ich mit einem Finger allein vermochte, d. h. ich drückte $4 \mathrm{mal}$ so oft den Schlüssel nieder.

Die beigefügten Kurven 1 and 2 illustriren das Gesagte:

Stimmgabel-Schwingungen 100 in einer Secunde.

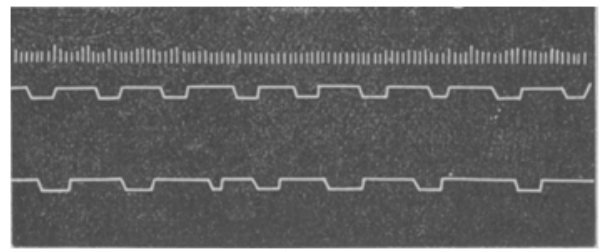

1. Morse-Schlüssel mit dem Zeigefinger möglichst oft herabgedrückt.

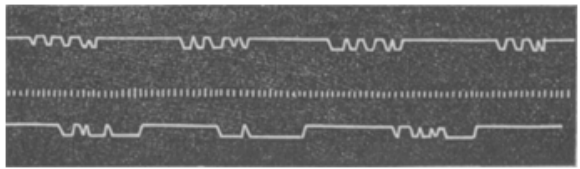

2. Morse-Schlüssel mit dem fünften bis zweiten Finger periodenweise möglichst schnell herabgedräckt.

Wir wollen jetzt festhalten, dass die Zahl der einfachen WillensHandlungen bei angestrengter Thätigkeit eine merkwürdige Uebereinstimmung mit der Zahl der rhythmischen Schwankungen des Tetanus und der häufigsten Tremorarten zeigt.

Nach den Auseinandersetzungen von Musterberg, Ziehen und Anderen besteht das Wesen der willkürlichen Handlungen darin, dass der 
Bewegung jedesmal eine Bewegungs-Vorstellung vorausgeht. Die Localisation der Bewegungs-Vorstellung verlegt man in die Rinde. Es folgt daher aus unseren Versuchen, dass - wenigstens die geprüften - Rindenganglien ebensoviel Impulse auszusenden vermögen, wio die untergeordneten Neurone.

Da wir nun einmal die ganze Betrachtung angestellt haben, um uns in die Lehre vom Tremor zu vertiefen, so dürfen wir einen wesentlichen Unterschied $\mathrm{z}$ wischen seinem Rhythmus und demjenigen der möglichst schnell wiederholten willkürlichen Bewegung nicht übersehen: Der Tremor beharrt lange auf seinem Rhythmus, obne dureb Ermüdung beeinträchtigt zu werden, die Willkür-Bewegung erreicht ibre höchste Ziffer nur vorübergehend. Man darf allerdings nicht vergessen, dass der von uns benutzten WillkürBewegung ein bedeutend grösserer Unfang zukommt, als gemeinhin den Zitterbewegungen, und dass dies, zum Theil wenigstens, die geringere A usdauer jener erk]ärt.

Es geht also - um das eben Besprochene noch einmal zusammenzufassen - aus unseren Auseinandersetzungen hervor, dass die Schnelligkeit der Zitterschwankungen, welche auf den ersten Blick so krankhaft anmuthet, ihr physiologisches Analogon hat, dass der Wille dem Zittern darin gleichzukommen vermag, dass mithin die höheren Neurone sich auf dieselbe Reizfrequenz einstellen können, wie die niederen. An welche Stelle des rentralnerven-Systems wir mithin den Ursprung des Zitterns verlegen, die Höhe der Frequenz seiner Schwankungen darf uns insofern nicht pathologisch äunken, als sie das normale Können nicht übersteigt.

Ich suchte nun die Beobachtungen an Gesunden durch solche an Kranken zu ergănzen. Es wurden daher Zitternde daraufhin untersucht, ob bei ibnen in jedem einzelnen Falle der eben erörterte Parallelismus zwisehen ibrem Zittern und ihrer Willkür-Bewegung besteht. Das Zittern wurde mit Hilfe von Marey'sehen Trommeln aufgeschrieben, und hiernach wurde der Patient aufgefordert, so oft, wie er nur konnte, mit dem Zeigefinger den Morse-Sehlüssel niederzudrücken und dessen elektrisches Signal in Thätigkeit zu setzen.

Die grösste Schnelligkeit, welche in der Anfeinanderfolge der Bewegungen erreicht wurde - der Record der Leistangen - wurde unter Berücksichtigung der Mittelwerthe notirt und mit dem Rhythmus des Tremors verglichen.

Der Zweck der Untersuchungen war theils theoretischer, theils practischer Natur. Liess sich feststellen, dass die Willkür-Bewegungee bei langsamem Tremor entsprechend langsam, bei schnellem entsprechend schnell aufeinander folgen, so wäre ein neues Symptom gewonnen, das in zweifelhaften Fällen von gewölnnlich zu Zittern führenden Krankheiten verwerthet werden könnte, sobald das Zittern fehlt. Würde man andererseits finden, dass die Willkür-Bewegungen sich anders verhalten, $z$. B. langsamer wiederholen, als die 
einzelnen Zitterschwankungen, so spräche der Befund dafür, dass der Ursprung des Zitterns in den untergeordneten Neuronen zu suchen ist.

Sehen wir zu, was sich nun thatsächlich fand. Meine Beobachtungen erstrecken sich auf 18 Kranke. Von ibnen litten 7 an Paralysis agitans, 4 an multipler Sklerose, 3 an Neurasthenie, 2 an Morbus Basedowii und je 1 an Anilin-Vergiftung und traumatischer Neurose.

Bei allen untersuchten Personen zeigte sich eine auffallende Uebereinstimmung der beiden uns interessirenden Zahlen. Die Menge der in 1 Secunde möglichen Willkür-Bewegungen war meist dieselbe, wie die Zahl der Zitterschwankungen. Bezeichnen wir die Zahl der letzteren mit Z, die der Willkür-Bewegungen mit $W$, so waren in den Einzelfällen bei

\begin{tabular}{|c|c|c|}
\hline Paral & sis & agitans \\
\hline No. & $\mathrm{W}$. & Z \\
\hline 1. & 7 & 7 \\
\hline 2. & 7 & 7 \\
\hline 3. & 6 & 5 od. 6 \\
\hline 4. & 6 & 5 od: 6 \\
\hline 5. & 5 & 5 \\
\hline 6. & 5 & 4 od. 5 \\
\hline 7. & 5 & fehlt \\
\hline
\end{tabular}

Sklerosis multiplex:

No. W. Z.

1. 66

2. 56

3. 55

4. 6 fehlt

Neurasthenie:

No. W. $Z$.

1. 99

2. 910

3. $10 \quad 11$

Morbus Basedowii: Traumat. Neurose: Anilin-Intoxication:

No. W. Z.

1. 910

W. Z.

78 od. 9

W. Z.

2. 76

77

Manvergleichemit denangegebenen Zablen die nachstehenden Proben der Curven, aus denen sie abstrahirt sind.

Paralysis agitans:

Tremor

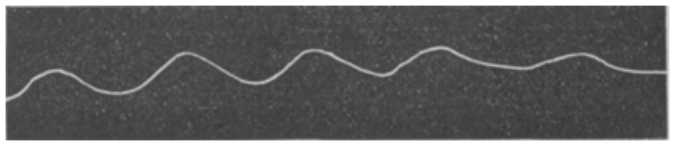

Wilkür-Bewegung.

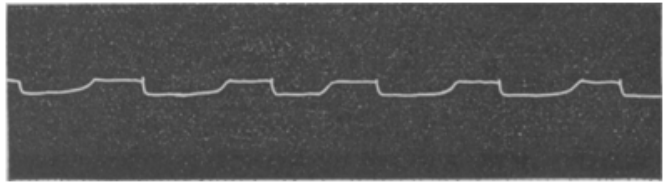


Maltiple Sklerose:

Tremor.

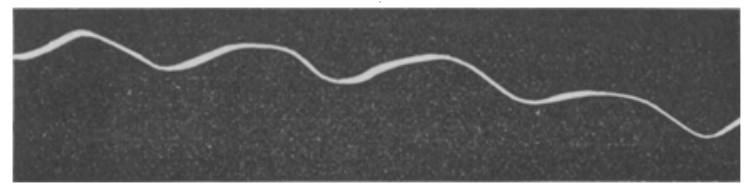

Willkür-Bewegung

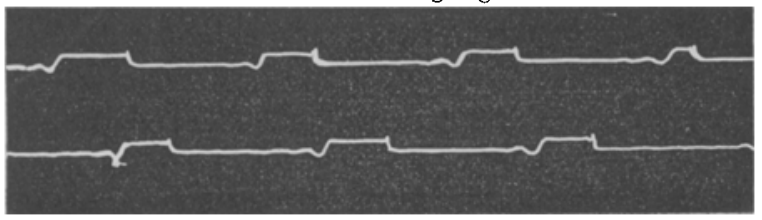

Neurasthenie:

Tremor

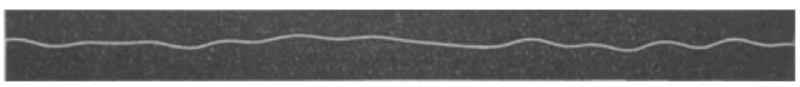

Willkür-Bewegung

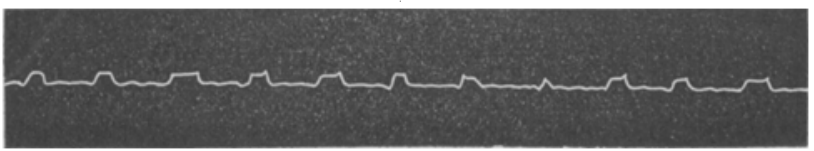

Morbus Basedowii:

Tremor

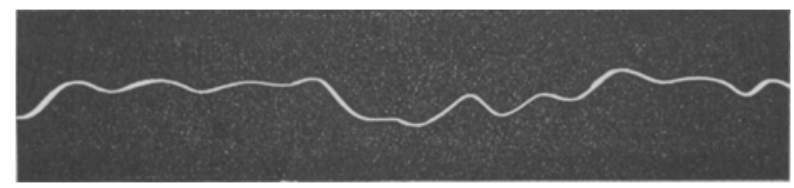

Willkür-Bewegung

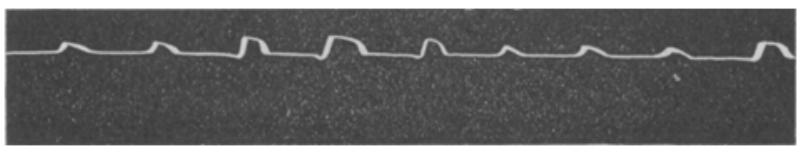

Traumatische Neurose:

Tremor

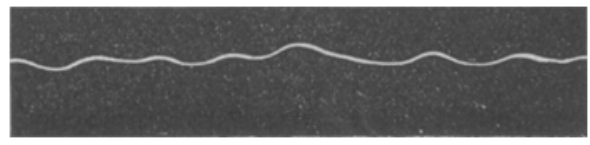




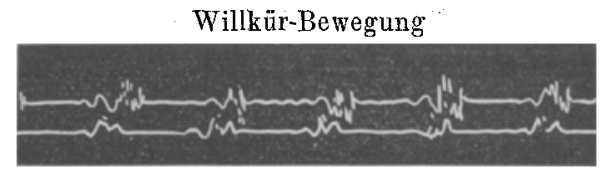

Anilin-Intoxication:

Tremor

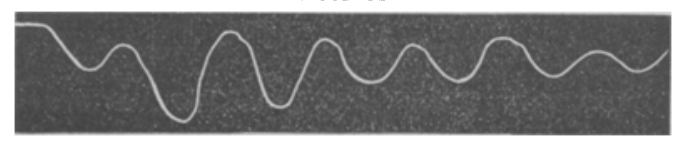

Willkür-Bewegung

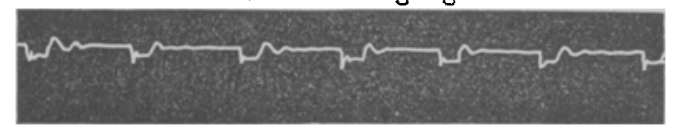

Die meisten Patienten verdanke ich der Poliklinik meines früheren Chefs Herrn Prof. Oppenheim ${ }^{1}$ ). Die Richtigkeit der Diagnose unterlag keinem Zweifel, und ich glaube daber der Pflicht enthoben zu sein, ermüdende Krankengeschichten mitzuteilen.

Ein Blick auf die Zahlen überzeugt uns, dass diejenigen der Zitterund Willkür-Bewegungen merkwürdig übereinstimmen. Wo z. B. die Zitterschwankungen in Anbetracht der vorhandenen Krankheit auffallend häufig waren, wie bei Paralysis agitans No.1 u. 2, war die Zahl der in 1 Secunde möglichen Willkür-Bewegụngen ebenfalls hoch; wo jene verhältnissmässig niedrig war, wie bei Morbus Basedowii No.2, war es W, ebenfalls. Ganz davon abgesehen zeigen die Krankheiten, welche gewöhnlich zu einem langsamen Rhythmus des Zitterns führen, also Paralysis agitans und multiple Sklerose, im Allgemeinen entsprechend geringe Werthe für die Schnelligkeit der Willkür-Bewegungen, und die Krankheiten mit raschem Tremor entsprechend. höhere.

Ein ganz besonderes Interesse verdienen die beiden Kranken, bei denen der Tremor fehlt, obwobl ihr Leiden gewöhnlich mit einem solchen verbunden ist. Der eine von ihnen litt an Paralysis agitans, der andere an multipler Sklerose.

Die Schüttellähmung betraf eine 56 jährige Frau. Das maskenartige Gesicht mit dem seltenen Lidschlag, die starre Körperhaltung, der vornübergeneigte Kopf, die eigenthümliche Stellung des Daumens, die Erschwerung der passiven, die Langsamkeit der activen Bewegungen offenbarten die Krankheit trotz des kaum angedeuteten Zitterns.

1) Ich benutze die Gelegenbeit, Herrn Prof. Oppenheim für die Ueberlassung der Patienten und den Herren Prof. J. M unk und Dr. Cowl für die technische Beihülfe meinen besten Dank zu sagen. 
Die multiple Sklerose, welche ein 25 jähriges Fräulein ergriffen hatte, war durch lebhafte Knie-Phänomene, sehr deutlichen Fuss-Klonus, motorische Schwäche des rechten Beines, vorübergehende Abducens-Lähmung charakterisirt.

Ich fand nun bei beiden Patienten, dass die Zahlen der ihnen in 1 Secunde möglichen Willkür-Bewegungen 5 und 6 betrugen, also im Vergleich mit den bei Gesunden festgestellten auffallend niedrig waren. Die Langsamkeit gilt aber auch als eines der wesentlichsten Charaktere des für diese Krankheiten eigenthümlichen Zitterns.

Die Beobachtungen sprechen also dafür, dass wir, ohne dass Zittern vorhanden ist, Aussicht haben, ein ihm in jener Beziehung parallel gehendes Phänomen nachzuweisen.

Zum Schlusse seien die wesentlichsten Resultate, für welche meine Versuche sprechen, eng zusammengestellt:

Die Hirnganglien sind făhig, in der Zeiteinheit ebensoviele Impulse auszusenden, wie die untergeordneten Centren.

Auch wenn wir die Ursache des Zitter-Rhythmus in die Hirnrinde verlegen, können wir uns auf ein physiologisches Analogon stützen.

Bei Zitternden entspricht die Anzahl der in der Zeiteinbeit möglichen Willkür-Bewegungen im Allgemeinen dem Zitter-Rhythmus. Wo das Zittern wider Erwarten fehlt, kann es - wenigstens in einer Reihe von Fällen in seiner diagnostisehen Bedeutung, soweit seine Frequenz in Frage kommt, durch Prüfung der Willkür-Bewegung ersetzt werden.

Endlich sei darauf aufmerksam gemacht, dass die von mir geäbte Methode ein Mittel an die Hand giebt, die Schwerfälligkeit activer Bewegungen, z. B. die der Schüttel-Lähmung, zahlenmässig zu beweisen.

\section{Berichtigung.}

Seite 278 Zeile 5 von oben muss es heissen: „Ineinandergefügtsein“ statt, „Ineingefügtsein ${ }^{\prime}$.

Seite 287 Zeile 14 von oben ist hinter "Korteweg“ einzufügen: "sagt".

J. Wolff. 